\title{
Topical Compounded Analgesic Treatment in Neuropathic Pain: 8 years of Experience
}

\author{
Jan M Keppel Hesselink ${ }^{1^{*}}$ and David J Kopsky ${ }^{2}$ \\ ${ }^{1}$ Institute for Neuropathic Pain, Spoorlaan 2a, 3735 MV, Bosch en Duin, Netherlands \\ 2Institute for Neuropathic Pain, Vespuccistraat 64-III, 1056 SN, Amsterdam, Netherlands
}

"Corresponding author: Jan M Keppel Hesselink, Institute for Neuropathic Pain, Spoorlaan 2a, 3735 MV, Bosch en Duin, The Netherlands, Tel: 31628671847, E-mail: info@neuropathie.nu

Received date: October 10, 2017, Accepted date: October 23, 2017, Published date: October 30, 2017

Copyright: @ 2017 Keppel Hesselink JM, et al. This is an open-access article distributed under the terms of the Creative Commons Attribution License, which permits unrestricted use, distribution, and reproduction in any medium, provided the original author and source are credited.

\begin{abstract}
In our tertiary referral centre, the Institute for Neuropathic Pain, we have treated many patients suffering from localized peripheral neuropathic pain since 2009. We have developed, together with a compounding pharmacist, a base cream as a topical formulation which has enabled us to compound creams based on amongst others amitriptyline, ketamine, clonidine, baclofen, and phenytoin. We have found that many patients profit from such topical formulations and we will describe our experiences using such creams over the last 8 years in this paper, since we first started exploring such topical treatments. We have analysed the efficacy and safety of these topicals, and based on literature, our own online data pool and the patients who had let us know that they benefited from the creams, found sufficient analgesia and positive effects impaired sleep, while the tolerability was excellent. Patients suffering from localized peripheral neuropathic pain, as in diabetic neuropathy, chronic idiopathic axonal polyneuropathy, chemotherapy induced polyneuropathy and small fiber neuropathy, as well as in complex regional pain syndrome, seem especially responsive to such topical approach. Meanwhile, more than 800 Dutch medical doctors have been prescribing one or more of our compounded creams, and patients and medical doctors from abroad frequently contact us to explore the therapeutic options for their patients based on these creams.
\end{abstract}

Keywords: Neuropathic pain; Diabetic neuropathy; Chemotherapy

\section{Introduction}

Neuropathic pain may be quite resistant to drug treatment. Efficacy is in general quite disappointingly modest, with low Numbers Needed to Treat (NNT). NNTs are between 6-7 for serotonin-noradrenaline reuptake inhibitors including duloxetine, between 7-8 for pregabalin, gabapentin and enacarbil, and around 10 for capsaicin highconcentration patches [1]. Clearly, there is much room for improvement. Furthermore, many patients complain about the central side effects such as dizziness, feeling zombie-like and other signs of cognitive impairment, because these in particular interfere with their quality of life.

We exclusively see patients suffering from peripheral neuropathic pain who are mostly referred to our tertiary referral center, the Institute for Neuropathic Pain, as non-responders to the classical (co-)analgesics by other pain-physicians, or who find us via our website (www.neuropahy.nl). Until 2008 our approach was based on rational polytherapy, which at that time seems to have the highest likelihood of success [2].

We also combined a variety of treatment modalities, such as percutaneous electrical nerve stimulation (PENS) and/or transcutaneous electrical nerve stimulation (TENS), together with various analgesics, which potentially allowed the use of lower and better-tolerated doses of such analgesics. This approach, however, was not always as successful as we wished and this provoked us to explore a different route: the development of topical formulations containing (co-)analgesics. This was because we felt such treatment approach would be devoid of systemic side effects in particular. Systemic side effects of current neuropathic pain medication tend to exclude elderly patients suffering from neuropathic pain [3].

At that time there were some indicators that topical treatments could indeed reduce pain, but the focus was mostly on topical agents such as lidocaine $5 \%$ patches and topical capsaicin [4]. The first treatment leads to anesthesia and the second one is basically a neuropathological treatment, inducting degeneration of small nerve fibers in the skin. Moreover, both approaches were not very popular due to the adverse events and cumbersome handling.

After exploring the literature, we found out that most studies using (co-)analgesics in topical formulations, such as amitriptyline and ketamine, explored a rather low dose of such Active Pharmaceutical Ingredients (APIs) [5]. Studies comparing the combination of amitriptyline $2 \%$ and ketamine $1 \%$ to placebo were found to be negative $[6,7]$. We felt that dose-ranging in topical studies has been conducted in a suboptimal way, and one should aim for much higher dose-ranges [8].

In this article, we will describe the development of our own line of thinking, which eventually led to various topical formulations we now use in our clinic, mainly based on phenytoin, baclofen, amitriptyline and ketamine. It is clear that more research will be required, especially randomized controlled trials (RCTs). However, as for most compounded creams there is prior art, and thus patenting is impossible. Finding financial resources for such studies has proven to be complicated, and therefore it will be difficult to penetrate guidelines. Thus, we expect topical analgesics will need more support from a practice-based platform, rather than be formally based on sufficient RCTs of good quality, including sufficient patients. In order to at least partially compensate for this problem, we developed a single-blinded 
response test. Physicians can test within 30 minutes, based on a comparison between placebo cream and active cream, whether a patient is a responder or non-responder to the active cream. We described this single-blind response test with the placebo cream and the active cream in more detail in a separate paper [9].

\section{Patient number 1: Diabetic neuropathic pain treated with a topical mix of 3 compounds}

In 2009 we saw a 62-years-old man suffering from intractable neuropathic pains in both feet and hands since 2003 [10]. The diagnosis was painful diabetic polyneuropathy. He scored 6 on the Douleur Neuropathique 4 questionnaire (DN-4; total score 10), which corresponds to severe neuropathic pain. When we first saw the patient his treatment consisted of pregabalin $75 \mathrm{mg}$ daily and topical capsaicin $0.075 \%$ cream. Neither of these medications had any effect on the pain. Because of the pain, his function was severely compromised. The patient described his pain as burning and excruciating. On the dorsal side of his right foot he experienced a painful feeling as if a clamp was squeezing his foot ("cramp pain"). Walking barefoot and sitting increased the pain. The symptoms worsened as the day progressed. Because of severe flatulence, a known side effect of pregabalin, the patient stopped using this drug. PENS once a week provided only little pain relief. TENS twice daily, Flos Cannabis in the evening, amitriptyline $20 \mathrm{mg}$ daily, and alpha lipoic acid $600 \mathrm{mg}$ daily slightly relieved his symptoms but without adequate analgesia (Figure 1).

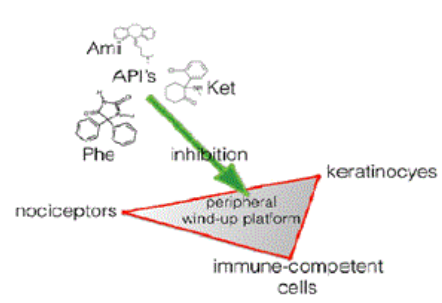

Figure 1: Active Pharmaceutical Ingredients (API), such as phenytoin (Phe), Ketamine (Ket) and Amitriptyline (Ami), brought in a suitable topical formulation can down-regulate peripheral sensitization in peripheral neuropathic pain via various targets in the skin and their cross-talk.

As the patient did not respond to our rational polytherapy, we prescribed a novel topical cream that we had developed in a neutral cream base, consisting of isosorbide dinitrate $0.4 \%$, capsaicin $0.075 \%$, and lidocaine $3 \%$. The cream was applied in amounts of about 1 gram per application, three times daily, as a thin layer on both soles. The pain intensity decreased by $50 \%$, and the cramping pain in his right foot disappeared. We developed this cream, consisting of three different drugs, based on three different mechanisms of action on the idea that multi-target approach in neuropathic pain would lead to pain reduction, in line with our general approach, and we introduced this first step as 'cream-enriched polytherapy'.

The success of this case provoked us to subsequently develop a line of various creams, and we continued to explore amitriptyline cream. We also developed a hypothesis of why topical analgesics would work without systemic resorption, based on 3 different targets in the epidermis: the small nerve endings (nociceptors), the keratinocytes, and the immune-competent cells [11]. All of these cell populations are characterized by a wide population of sodium channels in their membrane, and there is an intense cross-talk between these cells, leading to peripheral sensitization.

\section{Selecting (co-)analgesics for topical formulations}

Various factors can influence the analgesic effect of a topical formulation, including the many physicochemical characteristics of the drug selected, and the vehicle into which the drug is formulated. Without wanting to go too much into detail here, various physicochemical characteristics, such as molecular weight, melting point, the partition coefficient, the capacity factor, and aqueous saturation solubility, all play important roles [12]. For instance, in order to penetrate the outer skin layer, a compound needs to have lipophilic characteristics and a relatively low molecular weight; it needs to be a small molecule [8]. Furthermore, the compounds need to be soluble in the selected vehicle, which in turn needs to be smearable, non-sticky and not unpleasant for patients when they rub it in. Most contemporary compounded analgesics are formulated in Pluronic Lecithin Organogel (PLO), and this vehicle is notable for its bad smell and stickiness. With this in mind we developed a neutral cream, a cream which also enabled us to mix the base with high doses and various (co-)analgesics in order to optimize its efficacy.

\section{Amitriptyline 5\% and $10 \%$ topical treatment}

We started with amitriptyline as the compound is lipophilic, has a low molecular weight and was already known to have been formulated in topical creams, albeit in PLO gel, and in a low concentration (2\%) only. We started with amitriptyline $5 \%$ and soon increased its concentration to $10 \%$ [13]. We reported our experiences and reviewed the literature in 2012 [13]. We presented two chronic neuropathic pain patients, both with a positive response to topical amitriptyline treatment and an apparent dose-related effect as well as dose-related adverse effects. In both cases, $10 \%$ amitriptyline cream was more effective than $5 \%$. These first results were congruent with published clinical studies at that time, in which higher concentrations of topical amitriptyline (sometimes combined with other active drugs) had shown significantly greater pain reduction compared to placebo or lower doses. In one case, a complete reduction of pain was obtained within 20 minutes after the application of amitriptyline $10 \%$, without any reoccurrence of pain during the day of application. This was our first pointer that such topical approach would lead to a fast onset of relief. Interestingly, there is no systematic literature on the relevance of a fast onset of action and pain relief in the literature related to neuropathic pain treatment. Most oral therapies need many days to weeks before analgesic effects are perceived, and this of course induces compliance problems and early drop-outs. It is therefore of high importance to develop therapies with a quick onset of relief, and we think that such therapies can be found in compounded topicals.

Amitriptyline cream in our hands proved to be of use for treating localized neuropathic pain, for instance in diabetic patients, but there were some minor drawbacks: sometimes there were signs of systemic absorption especially in elderly patients with frail skins, and burning sensations limited its use in around $10 \%$ of patients. We therefore explored other options. First we developed 10\% ketamine and 5\% baclofen creams, and more recently $5 \%$ and $10 \%$ phenytoin creams. 


\section{Ketamine 10\%: Opening the therapeutic window to CRPS}

While exploring the therapeutic spectrum of amitriptyline $10 \%$ cream, we were faced with some limitations. For instance, complex regional pain syndrome (CRPS) is a painful disorder for which no therapy yet exists, and thus we started to explore the value of amitriptyline. However, after some initial responders, we soon felt the local side effects were too frequent for amitriptyline to be of use; many patients complained of burning side effects. This of course is not very helpful in an indication such as CRPS. Subsequently, with CRPS, we also felt amitriptyline did not have any relevant analgesic actions. We therefore explored a new (co-)analgesic, ketamine. We found ketamine cream to be of much more use in CPRS pains, especially since it abolished or reduced allodynia relatively quickly [14-16]. Ketamine ointment was already reported in 2002 to be of use in CRPS, but its greasiness probably limited its further use. Moreover, the selected dose at that time was minute, $0.25 \%-1.5 \%$ [17]. On the other hand, a double blind controlled study showed that ketamine $10 \%$ cream had significantly more pain reducing effect on allodynia in CRPS as compared to placebo cream [18].

In our experience, ketamine $10 \%$ cream in the selected neutral base cream leads to high compliance, and we did not see any indicators for systemic absorption. In 2011 we were able to publish our first results with a compounded ketamine cream, a successful treatment of a CRPS typel patient with severe intractable pain, following a multimodal stepped care approach, using creams of amitriptyline, ketamine, and dimethylsulphoxide (DMSO). As the first step of our treatment schedule we selected topical amitriptyline $5 \%$ cream. In the past our patients responded to some extent to oral amitriptyline; however, adverse effects limited its use. Therefore, topical amitriptyline was well justified but did not result in sufficient pain reduction. As a second step we added topical ketamine $10 \%$ cream. Ketamine acts on the Nmethyl-D-aspartic acid receptor (NMDAR), located on the small nerve fibers in the skin. The pain-reducing effect of ketamine cream we explained based on increased inflammatory factors are present in CRPS, and inflammation in the periphery increases the number of NMDAR on peripheral nerve fibers [19]. As a third step we added DMSO 50\% cream. DMSO acts as a penetration enhancer for other topically administered drugs, in particular amitriptyline and ketamine, both which we used [20].

Each step lead to a decrease of pain scores, from an initial 9 and eventually down to 1.5 . Since that initial patient we have treated more such patients with ketamine $10 \%$ and reported some of these cases in literature [15].

\section{Phenytoin 5\% and 10\%: Quicker action of onset and boosting effects}

In 2014 we started developing a topical cream formulation containing phenytoin or phenytoin sodium, as a standalone or in combination with other (co-)analgesic compounds. We chose phenytoin because we wanted to improve our results in various localized peripheral neuropathic pain states, and therefore turned to this 'mother of all anticonvulsants', synthesized in 1908 and first introduced in the clinic by Merritt and Putnam in 1937[21]. Phenytoin is one of the most remarkable multi-purpose drugs in literature, and has been repositioned since the 1940s in a plethora of indications [22].

Phenytoin has a number of mechanisms of action, making its use in a compounding analgesic cream likely, such as broad channel blocking properties, especially related to the family of sodium channels, as well as some anti-inflammatory and neuroprotective properties [23] (Figure 2).

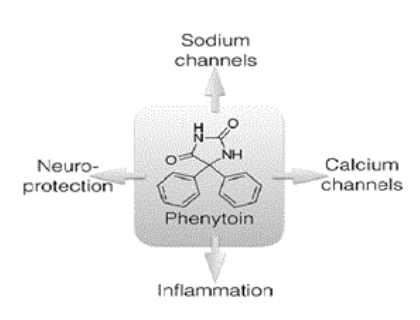

Figure 2: The 4 putative mechanisms of action of phenytoin as an analgesic in a topical cream: inhibition various ion channels, both sodium as well as calcium channels, anti-inflammation and neuroprotection.

Before we could use this cream in our clinic however, we had to solve a number of formulation issues, especially since phenytoin sodium leads to $\mathrm{pH}$ changes in the base cream. After this we found solutions in order for us to start testing the cream in a number of localized peripheral neuropathic disorders [24].

To date, we have described in detail the successes of over 70 patients treated with this cream, most of whom were suffering from pain from diabetes mellitus, chronic idiopathic polyneuropathy, chemotherapy induced polyneuropathy, small fiber neuropathy and post-herpetic neuralgia. We will publish the results of a pooled-analysis of these patients elsewhere. We also started publishing cases and case-series where we explored the clinical spectrum of phenytoin [25-27]. Our hypothesis is that those indications which are especially characterized by small fiber pathology in the skin and those indications where patients complain about burning pain in localized areas will be the indications leading to the highest likelihood of success.

Based on our clinical experience, as well as on the input we received from colleagues, it became clear for us that phenytoin cream has certain advantages over other (co-)analgesics, like the fast onset of relief within 20 minutes, the absence of systemic resorption (based on a plasma analysis in 16 patients), and a strong boosting effect when combined with other (co-)analgesics. As our findings were new and innovative we were lucky to be able to design 2 patents, thus making a worldwide development possible because now there is a clear incentive for exploring its safety and efficacy in RCTs.

\section{Conflict of Interest}

The authors are patent holders of two patents related to the topical formulations of phenytoin in the treatment of pain:

- Topical phenytoin for the use in the treatment of peripheral neuropathic pain, and

- Topical pharmaceutical composition containing phenytoin and a (co-)analgesic for the treatment of chronic pain.

\section{References}

1. Finnerup NB, Nadine A, Simon H, McNicol E, Ralf B, et al. (2015) Pharmacotherapy for neuropathic pain in adults: A systematic review and meta-analysis and updated NeuPSIG recommendations Lancet Neurol 14: $162-173$. 
2. Dobecki DA, Schocket SM, Wallace MS (2006) Update on pharmacotherapy guidelines for the treatment of neuropathic pain. Curr Pain Headache Rep 10: 185-90.

3. Sawynok J (2014) Topical analgesics for neuropathic pain in the elderly: Current and future prospects. Drugs Aging 31: 853-862.

4. Zin CS, Nissen LM, Smith MT, O'Callaghan JP, Moore BJ (2008) An update on the pharmacological management of post-herpetic neuralgia and painful diabetic neuropathy. CNS Drugs 22: 417-442.

5. Lynch ME, Clark AJ, Sawynok J, Sullivan MJ (2005) Topical amitriptyline and ketamine in neuropathic pain syndromes: An open-label study. J Pain 6: 644-649.

6. Lynch ME, Clark AJ, Sawynok J (2003) A pilot study examining topical amitriptyline, ketamine, and a combination of both in the treatment of neuropathic pain. Clin J Pain 19: 323-328.

7. Lynch ME, Clark AJ, Sawynok J, Sullivan MJ (2005) Topical 2\% amitriptyline and $1 \%$ ketamine in neuropathic pain syndromes: A randomized, double-blind, placebo-controlled trial. Anesthesiology 103 140-146.

8. Hesselink KJM, Kopsky DJ, Stahl SM (2017) Bottlenecks in the development of topical analgesics: Molecule, formulation, dose finding and phase III design. J Pain Res 10: 635-641.

9. Hesselink KJM, Kopsky DJ (2017) Burning pain in small fibre neuropathy treated with topical phenytoin: Rationale and case presentations. J Clin Anesth Pain Med 1: 1-6.

10. Kopsky DJ, Keppel Hesselink JM (2010) A new combination cream for the treatment of severe neuropathic pain. J Pain Symptom Manage 39: e9e10.

11. Hesselink KJM, Kopsky DJ, Bhaskar A (2016) Skin matters! The role of keratinocytes in nociception: A rational argument for the development of topical analgesics. J Pain Res 10: 1-8.

12. Liu KS, Huang TH, Aljuffali IA, Chen EL, Wang JJ, et al. (2017) Exploring the structure-permeation relationship of topical tricyclic antidepressants used for skin analgesia. Int J Pharm 523: 386-397.

13. Kopsky DJ, Hesselink JM (2012) High doses of topical amitriptyline in neuropathic pain: Two cases and literature review. Pain Pract 12: 148-153.

14. Kopsky DJ, Hesselink KJM (2011) Multimodal stepped care approach involving topical analgesics for severe intractable neuropathic pain in CRPS Type 1: A Case Report. Case Rep Med 2011: 319750.
15. Hesselink KJM, Kopsky DJ (2013) Treatment of chronic regional pain syndrome type 1 with palmitoylethanolamide and topical ketamine cream: Modulation of nonneuronal cells. J Pain Res 6: 239-245.

16. Kopsky DJ, Hesselink KJM, Bhaskar A, Hariton G, Romanenko V, et al. (2015) Analgesic effects of topical ketamine. Minerva Anestesiol 81: 440-449.

17. Ushida T, Tani T, Kanbara T, Zinchuk VS, Kawasaki M, et al. (2002) Analgesic effects of ketamine ointment in patients with complex regional pain syndrome type 1. Reg Anesth Pain Med 27: 524-528.

18. Finch PM, Knudsen L, Drummond PD (2009) Reduction of allodynia in patients with complex regional pain syndrome: A double-blind placebocontrolled trial of topical ketamine. Pain 146: 18-25.

19. Carlton SM, Coggeshall RE (1999) Inflammation-induced changes in peripheral glutamate receptor populations. Brain Res 820: 63-70.

20. Gurtovenko AA, Anwar J (2007) Modulating the structure and properties of cell membranes: The molecular mechanism of action of dimethyl sulfoxide. J Phys Chem B 111: 10453-10460.

21. Keppel Hesselink JM, Kopsky DJ (2017) Phenytoin: 80 years young, from epilepsy to breast cancer, a remarkable molecule with multiple modes of action. J Neurol 264: 1617-1621.

22. Keppel Hesselink JM (2017) Amantadine and phenytoin: Patent protected cases of drug repositioning. Clin Invest 7: 11-16.

23. Keppel Hesselink JM (2017) Phenytoin: A step by step insight into its multiple mechanisms of action-80 years of mechanistic studies in neuropharmacology. J Neurol 264: 2043-2047.

24. Kopsky DJ, Keppel Hesselink JM (2017) Topical phenytoin for the treatment of neuropathic pain. J Pain Res 10: 469-473.

25. Hesselink KJM, Kopsky DJ (2017) Topical phenytoin cream reduces burning pain due to small fiber neuropathy in sarcoidosis. J Anesth Pain Med 2: 1-3.

26. Kopsky DJ, Keppel Hesselink JM (2017) Phenytoin in topical formulations augments pain reduction of other topically applied analgesics in the treatment of trigeminal neuralgia. J Clin Anesth 38: 154-155.

27. Keppel Hesselink JM, Kopsky DJ (2017) Topical phenytoin in neuralgic pain, peripheral modulation of central sensitization: Two case reports. J Pain Relief 6: 1-5. 